Objectives: To address this need and our hypothesis that age-related immune effector and regulatory cell subsets changes are present, we employ a multidimensional approach using mass cytometry to identify the cell subsets that shape the developing immune landscape from birth to adulthood.

Methods: We interrogated the peripheral blood mononuclear cells from 126 healthy individuals (cord blood, newborn to adult) with mass cytometry using two extensive antibody panels embracing the most important cell lineages and their function. Quality control and batch effect correction were performed with outliers excluded before dimensional reduction and clustering to identify the unique immune cell subsets. Their frequencies across the age categories were presented as 3-D frequency histograms. Subsequent manual gating was done to further describe these immune cell subsets changes with age using Pearson correlation coefficient.

Results: Distinct developmental gradients involving multiple effector and regulatory cell subsets shaped the maturing immune landscape. The naïve TNF $\alpha+C D 4$ $+T$ cells were enriched in the early childhood period and declined with age (Pearson correlation coefficient, $r=-0.3503, p<0.001)$. In contrast, the memory TNF $\alpha$ $+C D 4+T$ cells increased with age $(r=0.4149, p<0.0001)$. A transitional milestone from naive to memory TNF $\alpha+C D 4+T$ cells was observed after 2 year old. This indicates that an intact CD4 +T cells TNF $\alpha$ effector mechanism is present at birth which progressively mature to involve the memory CD4 +T cells. For another cytokine IL17A, it was secreted solely by the memory CD4 $+\mathrm{T}$ cells with its population increasing with age $(r=0.3753, p<0.001)$. Another distinct maturation milestone was observed in the naive CD8 $+\mathrm{T}$ cell subset where a transition from IL8 to IFN $\gamma$ secretion after 10 year old was observed. The IL $8+$ and IFN $\gamma+$ naive CD8+T cell subsets correlated negatively and positively with increasing age respectively $(r=-0.2661, p<0.01$ and $r=0.2835, p<0.01)$.

For the $T$ regulatory cells, the age related changes were more gradual. The early thymic T regulatory cells (CD3+, CD4+, CD31+, CD45RA+, CD25+, Foxp3+, CD152+) demonstrated a gradual decline with age $(r=-0.2966, p<0.01)$ while the memory $T$ regulatory cells gradually increased with age (CD3+, CD4+, CD45RO ,+ CD25+, Foxp3+, CD152+) $(r=0.2444, p<0.05)$.

Conclusions: Key developmental milestones in the $T$ cell compartment were identified and with the other subsets identified, a holistic description of the developing immune landscape was obtained. This atlas has the potential dual translational role of defining the stage of immune maturity and distilling the pathological cell subset in both paediatric and adult immune mediated diseases. The database and the related pipeline to analyse it will be provided as a free reference.

Disclosure of Interest: None declared

DOI: 10.1136/annrheumdis-2018-eular.6099

\section{AB1194 ARE THERE NEW PARAMETERS TO BE CONSIDERED IN SPECTRAL DOPPLER TO EVALUATE THE NAIL BED IN PSORIASIS AND PSORIASIS ARTHRITIS?}

\section{J.A. Mendonça ${ }^{1}$, L.N. Pansani ${ }^{1}$, M.B. Mimoto ${ }^{1}$, E.C. Machado ${ }^{2}$, A.C. Bazan ${ }^{2}$. ${ }^{1}$ Rheumatology; ${ }^{2}$ Dermatology, PONTIFICAL CATHOLIC UNIVERSITY OF} CAMPINAS, Campinas, Brazil

Background: Other spectral Doppler parameters can assess joint impairment caused by psoriatic arthritis and psoriasis ${ }^{1}$.

Objectives: To detect and compare Doppler velocimetric indexes changes in 3 groups of patients.

Methods: Thirty - eight patients were evaluated: 8 in the control group - healthy (52 nail beds); 15 in the psoriasis group (134 nail beds); and 15 in the group with psoriatic arthritis (147 nail beds). "CASPAR" criteria were used to classify the patients. The ultrasound (US) was performed in all patients using Esaote MyLab 50 , with high resolution linear probe with a frequency of $18 \mathrm{mHz}$, it was positioned longitudinally to the nail bed.

Results: The psoriasis group of patients included 7 males (46.6\%) and 8 females (53.4\%); $66.6 \%$ were white, $33,4 \%$ black.

Abstract AB1194 - Table 1. Mean and standard deviations of US, clinical and laboratory variables by clinical group:

\begin{tabular}{lccc}
\hline & Psoriatic Arthritis & Psoriasis & Control \\
\hline $\mathbf{R I}($ Resistance Index) & $0.58(0.09)$ & $0.59(0.10)$ & $0.60(0.07)$ \\
$\mathbf{P I}($ Pulsatility Index) & $1.05(0.31)$ & $1.06(0.45)$ & $1.12(0.25)$ \\
Acceleration $\left(\mathbf{A}=\mathrm{m} / \mathrm{s}^{2} ;\right)$ & $0.37(0.29)$ & $0.39(0.47)$ & $0.51(0.44)$ \\
Acceleration Time $(\mathbf{A T}=\mathrm{ms})$ & $107.30(37.10)$ & $114.64(41.14)$ & $113.10(40.10)$ \\
Angle of Insonation & $18.21(25.8)$ & $14.76(24.71)$ & $27.28(30.10)$ \\
$\left(\boldsymbol{\alpha}={ }^{\circ}\right)$ & & & \\
Nail bed thickness & $1.60(0.78)$ & $1.68(0.50)$ & $1.44(0.40)$ \\
$(\mathbf{T}=\mathrm{mm})$ & & & \\
VHS & $27.5(24.9)$ & $20.4(14.9)$ & - \\
PCR & $0.75(1.1)$ & $0.54(0.7)$ & - \\
\hline
\end{tabular}

Abstract AB1194 - Table 2. Distribution and percentage of individuals according to clinical group and to the PASI, NAPSI variables:

\begin{tabular}{lccc}
\hline & Psoriatic Arthritis $\mathrm{N}(\%)$ & Psoriasis $\mathrm{N}(\%)$ & Control N $(\%)$ \\
\hline NAPSI & & & \\
Normal $(=0)$ & $0(0)$ & 0 & $8(100)$ \\
Mild $(<20)$ & $4(28,6)$ & $10\left(^{71,4}\right.$ & 0 \\
Severe $(>20)$ & $11\left({ }^{(68,7}\right.$ & $5(31,3)$ & 0 \\
PASI & & & 0 \\
Mild $(<15)$ & $14\left(^{51,8}\right.$ & $13\left(^{48,2}\right.$ & 0 \\
Moderate ${ }^{15-25}$ & $1(50,0)$ & $1(50,0)$ & 0 \\
Severe $(>25)$ & 0 & $1(100,0)$ & 0 \\
\hline
\end{tabular}

Statistically significant: Mean age and SD of the psoriasis, psoriatic arthritis and control groups, respectively: $57.13 \pm 14.3$ years; $54.66 \pm 10.6$ years; $24.87 \pm 2.03$ years $(p<0.05)$. A statistically significant difference was found in the NAPSI variable among all groups $(\mathbf{p}<0.05)$; in the variable PASI, a difference was only found in the control group $(\mathbf{p}<\mathbf{0 . 0 5})$ (Kruskall-Wallis) (table 2$)$; the A variable between the psoriasis group and control $(\mathbf{p}<\mathbf{0 . 0 0 1})$; between the psoriatic arthritis group and control $(\mathbf{p}=\mathbf{0 . 0 0 1})$, the variable $\mathbf{T}$ between the psoriasis and psoriatic arthritis groups $(p=0.006)$ and between the psoriasis and control groups $(p=0.001)$ and the $\alpha$ variable between control and psoriasis groups $(p=0.017)$ (table 1). Spearman and Pearson correlations between US variables per group, psoriasis, psoriatic arthritis and control were: RIXPI $r=0.744(p<0.001), P D$ (power Doppler) xT $r=0.301$ (p<0.001), RIxPI $r=0.914$ ( $p<0.001)$, PIxA $r=0.46(\mathbf{p}<0.001) ; R I x P I$ $r=0.889(p<0.001)$, PDxT $r=0.490(p<0.001)$ respectively.

Conclusions: There are other parameters in spectral Doppler to be validated in order to characterise changes in nail beds.

\section{REFERENCE:}

[1] Mendonça JA. High Specificity of Spectral Nail Assessment in Psoriatic Arthritis Patients [abstract]. Arthritis Rheumatol. 2015;67(suppl 10).

Disclosure of Interest: None declared

DOI: 10.1136/annrheumdis-2018-eular.3768

\section{AB1195 DEVELOPMENT AND VALIDATION OF AN ULTRASONOGRAPHIC ACTIVITY SCORE (USAS) FOR RHEUMATOID ARTHRITIS}

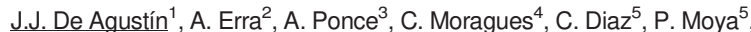
D. Reina ${ }^{6}$, P. Estrada 6 , E. Casado ${ }^{7}$, M. Moreno ${ }^{7}$, J. ramirez ${ }^{8}$, L. Mateo ${ }^{9}$, M. Pujol ${ }^{10}$, S. Ros ${ }^{11}$, P. Santo ${ }^{12}$, E.L. Sirvent ${ }^{12}$, J. Narvaez ${ }^{13}$, R. Sanmarti $1^{14}$, M. Garcia Yebenes ${ }^{15}$, L. Carmona ${ }^{15}$, on behalf of ECOCAT GROUP. ${ }^{1}$ Rheumatology Unit, Hospital Universitari Vall d'Hebron; ${ }^{2}$ Rheumatology, Hospital San Rafael, Barcelona; ${ }^{3}$ Rheumatology Unit, Hospital Granollers, Granollers; ${ }^{4}$ Rheumatology, Hospital Platon; ${ }^{5}$ Rheumatology, Hospital Sant Pau, Barcelona; ${ }^{6}$ Rheumatology, Hospital Moises Broggi, Sant Joan Despi; ${ }^{7}$ Rheumatology, Hospital Parc Tauli, Sabadell; ${ }^{8}$ Rheumatology, Hospital CLinic i Provincial, Barcelona; ${ }^{9}$ Rheumatology, Hospital Germans Trias i Pujol, Badalona; ${ }^{10}$ Rheumatology, Hospital de Tarrassa, Tarrassa; ${ }^{11}$ Rheumatology, Hospital de Viladecans, Viladecans; ${ }^{12}$ Rheumatology, Parc Sanitari Sant Joan de Deu, Sant Boi de Llobregat, ${ }^{13}$ Rheumatology, Hospital Universitari de Bellvitge, Hospitalet de Llobregat, ${ }^{14}$ Rheumatology, Hospital Clinic, BARCELONA; ${ }^{15}$ Inmusc, Instituto de Investigacion Musculoesqueletica, Madrid, Spain

Background: Composite scores developed in Rheumatoid arthritis (RA) not include all dimensions of disease activity. An index based on essential clinica plus a ultrasound (US) measures, focused on simplicity, with appropriate validation, would allow a better classification at different levels of disease activity than a clinical only or US only index.

Objectives: To develop and validate a mixed clinical-US inflammation score in RA for use in clinical practice.

Methods: Mixed methods. Experts elicited items reflecting inflammation which were prioritised by Delphi. Patients with RA with various grades of activity underwent clinical [28 swollen and tender joints counts, patient and physician globa assessment (PhGA), erythrocyte sedimentation rate, and $\mathrm{C}$-reactive protein (CRP)] and US assessments [synovitis or tenosynovitis by grey-scale (GS) and Power Doppler (PD) of 42 structures], blinded to the clinical assessment. An index was created after supported selection of US structures and scoring method. Construct validity was tested by correlation with DAS28, SDAI, CDAI, and PhGA. Reliability was evaluated in a subgroup of patients with the intraclass correlation coefficient (ICC).

Results: US of joints and tendons, CRP, and swollen joints were the items that passed the prioritisation phase. Then, 281 patients were randomly divided into design $(n=141)$ and validation analysis $(n=140)$. The combination of US sites chosen detected the maximum proportion of GS and PD present. Were elected 
wrist, 2-3 MCP, Knee, tibio-talar and 2-3 MTP joints, and the following tendons: carpal extensor and flexor tendons, tibial posterior and peroneal. For scoring structures, three methods were tested: semiquantitative (0-3 GS +0-3 PD), dichotomous (0/1 GS +0/1 PD), and qualitative ( $0 / 1$ based on algorithm [image 1]). All showed strong correlation with activity measures ( $r$ o $\geq 0.60$ ), and reliability (ICC 0.89 to 0.93). The most feasible index, qualitative, was chosen.

The proposed formula for USAS was: $U S A S=N^{\circ}$ swollen joints + US score+CRP

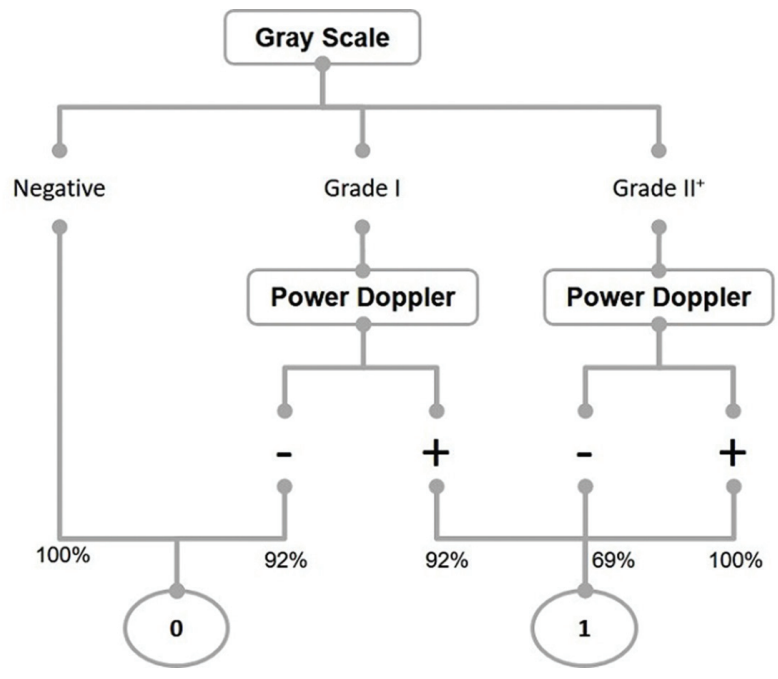

Abstract AB1195 - Figure 1

Conclusions: USAS is a valid and reliable measure of inflammation in RA equal to the sum of 28 swollen joint count, a simplified (0/1) US assessment of 11 structures and CRP.

Disclosure of Interest: None declared

DOI: 10.1136/annrheumdis-2018-eular.3824

\section{AB1196 ROLE OF CAPILLAROSCOPY IN THE STUDY OF THE RAYNAUD PHENOMENON IN CONDITIONS OF CLINICAL PRACTICE}

J. Uceda ${ }^{1}$, R. Hernandez ${ }^{1}$, C. Almeida ${ }^{2}$, J.L. Marenco ${ }^{1} .{ }^{1}$ Rheumatology;

${ }^{2}$ Estadistic, University Valme Hospital, Seville, Spain

Background: The Raynaud phenomenon (RP) is very common throughout the world, especially in cold climates, affecting approximately $3 \%-5 \%$ of the population. Most RPs are primary. The risk of the onset of autoimmune disease associated with RP (ADRP) is between $6 \%$ and $12 \%$, and the diagnosis is usually made within 2 years of its onset. Capillaroscopy is a simple, innocuous and inexpensive technique that is very useful in the study of RP

Objectives: The primary goal was to evaluate the capillaroscopic findings in patients with RF as the only symptom and establish their relationship between the subsequent development of ADRF.

Methods: We conducted an observational, descriptive, cross-sectional study of a random sample of 98 patients with RP as the main manifestation, selected from within the capillaroscopy consult of our Unit. The capillaroscope used was a stereomicroscope (Stereoscope), with a triocular head, zoom range from $1 x$ to $4 x$ magnification, with cold light illuminator and high resolution ocular camcorder. Periungual capillaroscopy was performed in 3rd, 4th and 5thfinger of the right and left hands in each patient and always by the same rheumatologist. We described the sociodemographic variables of patients, and analytical results of the antinuclear antibodies (ANA). After an initial statistical exploration of the data, the same are described. The quantitative variables are expressed as means and standard deviations and if the distributions are asymmetric with medians and quartiles. The qualitative variables with percentages. To assess the validity of capillaroscopy as a diagnostic test, we determined the sensitivity, specificity, and positive and negative predictive value of the test.

Results: $73.5 \%$ were women, with an average age of 45.7 years. The median time of evolution of the RP until the capillaroscopy was 2.5 years. $76 \%$ of the patients were ANA negative.
The evaluation of the predictive capacity of capillaroscopy for the development of EARP yields the following results:

Abstract AB1196 - Table 1

\begin{tabular}{lc}
\hline Sensibility & $83,3 \%{ }^{62,6-95,2}$ \\
\hline Specificity & $78 \%^{66,8-86,9}$ \\
Predictive positive value & $34,1 \%\left(^{24,5-45,3}\right.$ \\
Predictive negative value & $97,1 \%\left(^{93,3-98,8}\right.$ \\
\hline
\end{tabular}

When analysing the relationship between the presence of ANA and the association with ADRF we found that it was independent $(p=0.002)$ of the result of capillaroscopy, being the risk of developing the disease in a patient with positive ANA 8.5 times higher than in an ANA negative patient.

Conclusions: Capillaroscopy in patients with RP has a high predcitive negative value, which allows us to estimated, with high reliability, the association of this phenomenon with autoimmune disease in patients with normal capillaroscopic patterns. Given the results of our study, capillaroscopy should be protocol in the RP study

Disclosure of Interest: None declared

DOI: 10.1136/annrheumdis-2018-eular.5759

\section{AB1197 1 MULTIPARAMETRIC ANALYSIS OF CONNECTIVE TISSUE DISEASE SPECIFIC AUTOANTIBODIES USING A SPOT IMMUNOASSAY (SERASPOT $\left.{ }^{\circledR} A N A\right)$}

K. Conrad ${ }^{1}$, M. Rejzek ${ }^{1}$, M. Aringer ${ }^{2}$, S. Rudolph ${ }^{3}$, L. Unger ${ }^{4}$, K. Lüthke $^{5}$, A. Gräßler ${ }^{6}$, N. Röber ${ }^{1} .{ }^{1}$ Medical Faculty of the TU Dresden, Institute of Immunology; ${ }^{2}$ University Hospital Carl Gustav Carus Dresden, Department of Medicine III, Dresden; ${ }^{3}$ Immune Center Chemnitz, Chemnitz; ${ }^{4}$ Municipal Hospital Dresden-Friedrichstadt, Department of Medicine I; ${ }^{5}$ Medical Practice of Internal Medicine and Rheumatology, Dresden; ${ }^{6}$ Medical Practice Internal Medicine and Rheumatology, Pirna, Germany

Background: Because autoantibody profiling compared to single autoantibody determination is more relevant for diagnostics, differential diagnostics and even prognostics of the different kinds of connective tissue diseases (CTD), cost and time saving multiplex assays are more and more used in routine practice. ${ }^{1}$ Objectives: Evaluation of the diagnostic relevance of the SeraSpot ANA assay (Seramun Diagnostica GmbH, Heidesee, Germany), a novel spot immunoassay for multiplex analysis of the main connective tissue disease (CTD) specific autoantibodies (AABs) against dsDNA, histone, nucleosome, Scl-70, U1-RNP, Sm, PCNA, RibP, Ro52/TRIM21, Ro60, La/SS-B, CENP-B, Jo-1, PM/Scl-100, and $\mathrm{Ku}$ ) based on autoantigens immobilised in microtiter plates.

Methods: AAB profiles using the SeraSpot ANA assay were determined in sera of 381 patients with CTD and 202 apparently healthy individuals (AHI). The CTD patients comprises 105 SLE, 117 systemic sclerosis (SSc), 32 Sjögren's syndrome (SjS), 58 idiopathic inflammatory myopathies (IIM), 5 mixed connective tissue disease (MCTD), and 64 undifferentiated connective tissue disease patients (UCTD).

Results: At least one CTD associated AAB was positive in $88.2 \%$ of the tested CTD patients. A high diagnostic specificity for CTD above $95 \%$ compared to $\mathrm{AHI}$ were found for antibodies to dsDNA, RibP, Sm, Ro60, Ro52, CENP-B, Scl70, PM/ $\mathrm{Scl}-100, \mathrm{Ku}$ and Jo-1. Excluding low-titre reactivity, the specificity of U1-RNP, nucleosome, histone and La/SS-B antibodies was also very high (96.5\%-98\%) regarding CTD diagnosis. The highest specificities vs. AHI were found for antiSm, -Ro60, -RibP and -Jo1 antibodies (99.5\%), followed by anti-CENP-B (99\%), dsDNA (98,5\%), -Ku (98.5\%) and -Ro52 antibodies (98\%). Regarding SLE, 104 (99\%) were positive for SLE-associated AABs. Anti-dsDNA antibodies were most frequently found $(88.6 \%)$. The highest specificities $(98.5 \%-99.5 \%)$ for SLE compared to AHI were found for anti-dsDNA, -RibP, -Sm, and -Ro60 antibodies. SjS relevant AABs against Ro60, Ro52 and La/SS-B were seen in $81.3 \%, 84.4 \%$ and $46.9 \%$ of the SjS patients, respectively. The diagnostic specificity of Ro60 antibodies for SLE and SjS compared to other SARD (excluding UCTD) is $96.8 \%$ and 99.5\%-100\% compared to AHI. SSc associated AAB against Scl-70, CENP-B, $\mathrm{PM} / \mathrm{Scl}-100$ and $-\mathrm{U} 1-\mathrm{RNP}$ were found in $53.0 \%, 20.5 \%, 8.6 \%$, and $13.7 \%$ of the included SSc cases, respectively, with diagnostic specificities between $96 \%$ and 99\%. AABs against Jo-1, PM/Scl-100, U1-RNP, Ro52 and Ku were positive with high specificity $(98.4 \%-99.5 \%$ ) in $29.3 \%, 10.3 \%, 19 \%$ (100\% of MCTD), $27.6 \%$ and $10.3 \%$ of IIM patients, respectively. 